Proceedings

\title{
Examining the Possibility of Improving the Properties of Sunflower Oil in Order to Obtain a Better Medium for the Process of Frying Food ${ }^{+}$
}

\author{
Ranko Romanić *, Tanja Lužaić and Ksenija Grgić
}

Citation: Romanić, R.; Lužaić, T.;

Grgić, K. Examining the Possibility of Improving the Properties of Sunflower Oil in Order to Obtain a Better Medium for the Process of Frying Food. Proceedings 2021, 70, 104. https://doi.org/10.3390/ foods_2020-07748

Published: 10 November 2020

Publisher's Note: MDPI stays neutral with regard to jurisdictional claims in published maps and institutional affiliations.

Copyright: $\odot 2020$ by the authors. Licensee MDPI, Basel, Switzerland. This article is an open access article distributed under the terms and conditions of the Creative Commons Attribution (CC BY) license (http://creativecommons.org/licenses/by/4.0/).
Faculty of Technology Novi Sad, University of Novi Sad, Bulevar cara Lazara 1, 21000 Novi Sad, Serbia; tanja.luzaic@tf.uns.ac.rs (T.L.); xxenija013@gmail.com (K.G.)

* Correspondence: rankor@uns.ac.rs; Tel.: +381-21-485-3700

+ Presented at the 1st International Electronic Conference on Food Science and Functional Foods, 10-25 November 2020; Available online: https://foods_2020.sciforum.net/.

\begin{abstract}
With the modern and accelerated way of life, frying has become an extremely common way of food preparation. In the frying process, hot oil or fat serves as a heat transfer medium. Ideal fat in all frying processes does not exist due to differences in the chemical composition of the product, process conditions, expected nutritive value, and shelf life of the final product. During frying, physical and chemical changes simultaneously occur changing the chemical composition of edible oils. The food is immersed in hot fat, in the presence of air, where the frying medium is directly affected by three agents: moisture from the food, atmospheric oxygen, and high temperature. Reactions that occur are hydrolysis, auto-oxidation, thermal oxidation, and thermal decomposition, and the products that occur affect various physical and chemical changes in fats, as well as in fried food. The quality and oxidative stability of vegetable oils or their resistance to changes caused by oxidative processes is the time during which oils can be protected from the (auto) oxidation process. Analytical methods used in practice to determine the oxidative stability of oils are accelerated oil oxidation test (Rancimat test and OSI index) and Schaal oven test. In this paper, the possibilities of improving refined sunflower oil in order to obtain oil with greater applications in the food frying process are examined. Standard refined sunflower oil, sunflower oil with altered fatty acid composition, as well as sunflower oil enriched with natural and synthetic antioxidants were tested. The obtained results were compared with palmolein, commonly used for food frying. Of the tested sunflower oils, high-oleic sunflower oil with an iodine value (IV) of $85 \mathrm{~g} / 100 \mathrm{~g}$, OSI index of 9.3, and total oxidation (TOTOX) index of 4.73 , increased 6.66 times after exposure to frying proving to be the most similar to palmolein (IV = $57 \mathrm{~g} / 100 \mathrm{~g}$; OSI = 17.8; TOTOX = 7.60).
\end{abstract}

Keywords: frying; sunflower oil; synthetic antioxidants; natural antioxidants; iodine value; OSI; TOTOX

\section{Introduction}

Deep frying is widely used to prepare many types of food around the world. High temperatures during food frying lead to complex levels of reactions that result in hydrolysis, oxidation, or polymerization of the oil. Hydroperoxides are the primary products of lipid oxidation, so peroxide determination can be used as an oxidation index in the early stages of lipid oxidation. During deep frying of oil, secondary products are formed, which are obtained by oxidative decomposition, such as esters, aldehydes, alcohol, ketones, lactones, and hydrocarbons. These secondary products negatively affect the taste, aroma, nutritional value, as well as overall quality of the fried food [1]. In addition, certain oxidation products are potentially toxic at relatively low concentrations [2]. Reactive oxygen 
species play an important role in lipid oxidation, which leads to the formation of cardiovascular disorders and cell damage [3].

Due to its presence in the diet, sunflower oil is often a frying medium. The composition of sunflower oil varies depending on the hybrid, environmental conditions, harvest, and processing. Oils rich in polyunsaturated fatty acids, namely sunflower oil, are particularly susceptible to oxidation under high-temperature conditions. In the frying process, the oxidation of polyunsaturated fatty acids can produce peroxides, followed by unconjugated double bonds in unsaturated fatty acids that undergo rearrangement and conjugated dienes are formed which are absorbed at $232 \mathrm{~nm}$. However, when oxidation of polyunsaturated fatty acids containing three or more double bonds occurs, conjugation can be prolonged by the formation of another double bond leading to the formation of conjugated trienes, which absorb light at $270 \mathrm{~nm}$. Thus, changes in UV absorbances are considered an effective indicator of the oxidation of polyunsaturated fatty acids [4]. To increase the stability of the oil, antioxidants can be added in the aim to disrupt the mechanism of free radicals, which lead to its decomposition.

To prevent lipid peroxidation in oils, synthetic antioxidants have been used as food additives for more than 50 years. The addition of synthetic antioxidants such as butylhydroxyanisole (BHA), butylhydroxytoluene (BHT), tert-butylhydroquinone (TBHQ), is one of the most effective and popular methods to prevent oxidation and changes in the sensory properties of the oil. However, there are growing concerns about the safety and health risks associated with the use of synthetic antioxidants, but increasing attention is focused on the utilization of biologically active substances derived from plants, which have antioxidant effects [5].

Oil refining aims to remove impurities and harmful compounds from crude, unrefined oil, thus increasing the stability and quality of the vegetable oils. However, after refining, compounds such as phospholipids, free fatty acids, pigments, metals, and moisture can be found in the oil in traces, and in the presence of environmental factors, they create oxidation reactions leading to the destruction of essential fatty acids, the formation of compounds with unpleasant taste and odor, as well as toxic compounds that can adversely affect human health. The oxidative stability of the oil can be improved by using antioxidants. Antioxidants enhance oxidative stability by removing free radicals and reactive oxygen species, inactivating peroxides, chelating metal ions, removing secondary oxidation products, and inhibiting prooxidative enzymes. Based on their mode of action, they can be classified into primary and secondary antioxidants [6]. Primary antioxidants break the chain reaction by reacting with lipid radicals and creating stable products. Primary antioxidants include phenols, vitamins, minerals, flavonoids, carotenoids, lycopenes, diterpenes, cumin, and their derivatives. Secondary antioxidants prevent or slow down oxidation by suppressing oxidation promoters, including metal ions and singlet oxygen, prooxidative enzymes, and other oxidants. These are compounds that destroy already created free radicals. Secondary antioxidants include butylhydroxyanisole (BHA), butylhydroxytoluene (BHT), tert-butylhydroquinone (TBHQ), and propyl gallate (PG).

The importance of replacing synthetic antioxidants with natural components obtained from various plants has greatly increased. Antioxidant action has been found in some components isolated from fruits and vegetables, which is as effective as the use of synthetic antioxidants. Plants are a rich source of phenolic compounds such as phenols, flavonoids, and anthocyanins. In addition to antioxidant activity, these components also affect the sensory characteristics of food [2]. Bioactive components, such as phenols and flavonoids, tend to remove free radicals and are suitable for use as antioxidants. The main identified natural antioxidants of plant origin include tocopherols, carotenoids, vitamin $\mathrm{C}$, and phenols [3].

Since oxidation takes place in different ways, under the influence of external (light, temperature) and internal (degrees of saturation, free fatty acids, fasfolipids, pigments, 
etc.) factors and due to different properties for each oil, there can be no adequate antioxidant to meets all expectations and equally affects different types of oils. Therefore, various researches are continuously performed in order to find the most effective antioxidant [6].

The aim of this paper is to investigate new possibilities of improving refined sunflower oil in order to obtain oil with widespread applications in the food frying process. Three refined sunflower oils were investigated (with synthetic antioxidant added, with natural antioxidant added, and high oleic refined sunflower oil) as well as palmolein and compared with the standard (linoleic) refined sunflower oil. Iodine value was determined with the aim to investigate the degree of unsaturation impact on oxidative characteristics of the oil. As oxidative characteristics indicators, the overall oil stability index (OSI) and total oxidation index (TOTOX) were examined. All investigations were done in the initial samples and after the frying process.

\section{Material and Methods}

\subsection{Material}

Standard (linoleic) refined sunflower oil, high oleic sunflower oil, and palmolein used in the study were supplied by an edible oil factory in Serbia or were purchased in a store on the local market. Added synthetic antioxidant (TBHQ) and natural antioxidant (rosemary extract) (Sigma-Aldrich, St. Louis, MO, USA) were purchased from Kefo (Zemun, Serbia a.d.), Belgrade and were added to the refined (linoleic) sunflower oil in the ratio $0.02 \%(w / w)$ or $200 \mathrm{ppm}$. The oils and antioxidants mixed with a magnetic stirrer $(50-60 \mathrm{~Hz})$ for 2 min and immediately transferred to 2 L PET bottles, which were completely filled with oil (no empty space), closed with the original plastic seal, and stored in the refrigerator at $0-4{ }^{\circ} \mathrm{C}$. Samples were marked as shown in Table 1.

Table 1. Samples used in this study.

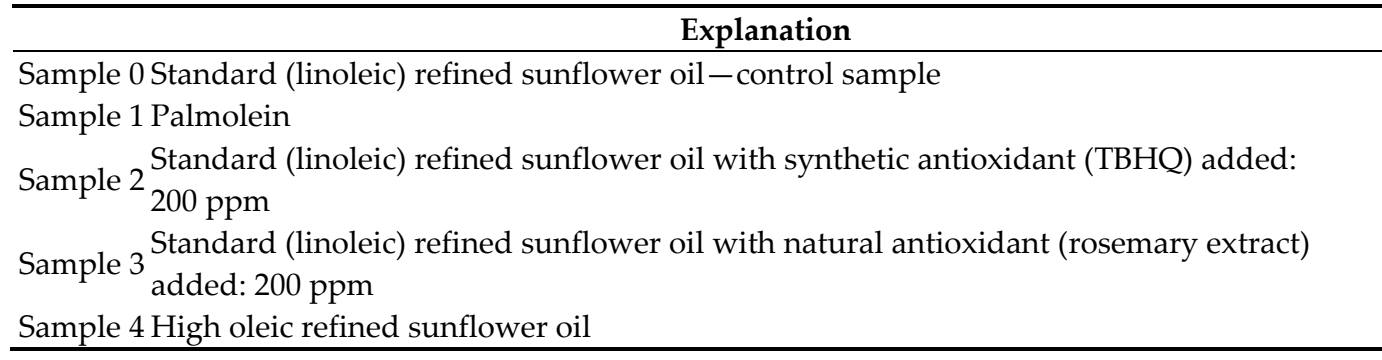

Oil samples were subjected to deep frying. Namely, the samples were used for frying French fries which were purchased at the local market. French fries $(600 \mathrm{~g})$ were fried in a controlled temperature domestic deep fat fryer model FF230831 (Tefal, UK) filled with 1.2 $\mathrm{L}$ of each oil sample used in this study. Frying conditions were $2.5 \mathrm{~min}$ at temperature $175^{\circ} \mathrm{C}$. After cooling, a portion of each oil after frying was taken for testing.

\subsection{Methods}

Iodine value, oxidative oil stability index (OSI), and total oxidation index (TOTOX) were investigated in the initial oil samples and oil samples after frying.

Iodine value was determined by iodometric titration according to ISO 3960: 2017 [7].

The oxidative oil stability index (OSI), measured as induction period (IP), was determined as described by Metrohm Application Bulletin 204/2e [8] and according to ISO 6886:2016 [9]. Rancimat apparatus, model 743 (Metrohm, Switzerland) at a temperature of $110{ }^{\circ} \mathrm{C}$ and an airflow of $20 \mathrm{~L} \mathrm{~h}^{-1}$ was used. The time that elapses between the beginning of the process and the appearance of the secondary reaction products is the oxidation induction period or oil stability index (OSI). Portions of oil (2-2.5 g) were weighed in each reaction vessel and analyzed simultaneously. The IP was determined automatically by the device and expressed as hours. 
The total oxidation (TOTOX) index was computed based on the peroxide value (PV) and anisidine value ( $p-\mathrm{AnV})$ using Equation (1) [10]:

$$
\text { TOTOX }=2 \times P V+p-A n V
$$

Peroxide values were determined by iodometric titration according to ISO 3960:2017 [11], while anisidine values spectrophotometric with a $p$-anisidine reagent were measured using a UV-VIS spectrophotometer (PG Instruments Ltd., Lutterworth, UK) in a $1 \mathrm{~cm}$ layer cuvette at 350 according to ISO 6885:2016 [12]. All used chemicals and reagents were of analytical grade (Sigma-Aldrich, St. Louis, MO, USA and J.T. Baker Avantor, The Netherlands).

All determinations were carried out in triplicate. The obtained data were subjected to analysis of variance (one way-ANOVA) with Tukey's HSD test used to study the significant differences at the 0.05 level. Statistical processing of the obtained results was performed using Statistica version 13.5.0.17 (StatSoft, Tulsa, OK, USA). The Pearson correlation coefficient was used to determine the correlation between OSI index, TOTOX index, and iodine value.

\section{Results and Discussion}

The iodine value indicates the degree of unsaturation of fatty acids. A decrease in iodine value shows a decrease in the number of double bonds and it indicates oxidation of the oil [13]. Samples 1 and 3 represent standard (linoleic) sunflower with added synthetic antioxidant (sample 1) and natural antioxidant (sample 3), while sample 0 represents a control sample (standard (linoleic) sunflower oil without additives). The volumetrically determined iodine values of samples 0,1 , and 3 do not differ statistically significantly $(p<0.05)$ and amounted to $122.69 \pm 3.21,122.87 \pm 4.23$, and $122.74 \pm 3.56 \mathrm{~g} / 100 \mathrm{~g}$, respectively. The obtained results are in line with expectations since the basis of these samples is the same oil. Significantly lower $(p<0.05)$ iodine values were determined in samples 4 and 2 and were $85.25 \pm 1.26$ and $57.28 \pm 0.87 \mathrm{~g} / 100 \mathrm{~g}$, respectively. These two samples also differ statistically significantly since sample 4 represents high oleic sunflower oil, while sample 3 is palmolein. The iodine values of samples after frying were also determined and no significant difference $(p<0.05)$ was found between the initial samples (Figure 1a) and the samples after frying (Figure 1b). The obtained results are in line with previous findings. Chebet et al. [13] tested the iodine values of sunflower and palm oil of the initial samples (126.9 \pm 1.67 and $50.8 \pm 0.72 \mathrm{~g} / 100 \mathrm{~g}$, respectively) as well as after the frying of chips twice, and obtained the following values: $121.8 \pm 2.45 \mathrm{~g} / 100 \mathrm{~g}$ (sunflower oil) and $45.1 \pm 2.29 \mathrm{~g} / 100 \mathrm{~g}$ (palmolein).

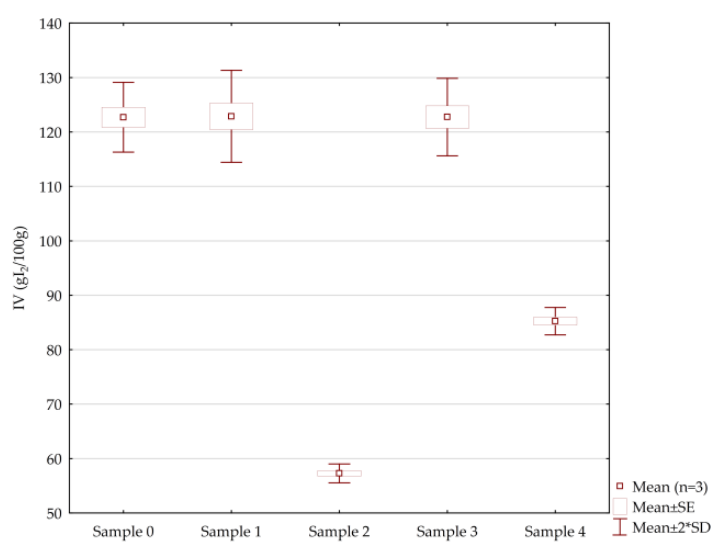

(a)

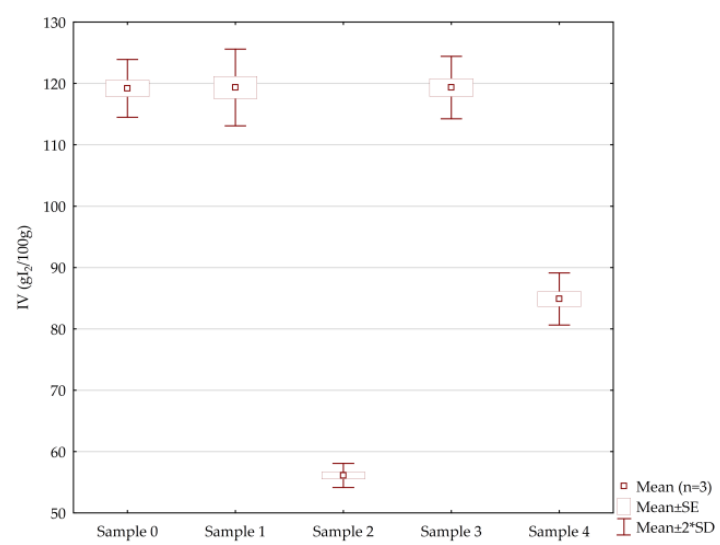

(b)

Figure 1. Box plot presented iodine values of (a) the examined initial samples and (b) examined samples after the frying process. 
The OSI index indicates the ability of an oil to resist oxidative changes [14,15]. The most oxidatively stable oils have the highest value of the OSI index. Sample 2 had the highest determined OSI index value of $17.82 \pm 1.01 \mathrm{~h}$, a significantly $(p<0.05)$ lower value was determined in sample $4(9.26 \pm 1.02 \mathrm{~h})$, while even lower values $3.98 \pm 0.41,3.97 \pm 0.45$, and $3.95 \pm 0.75 \mathrm{~h}$ were determined in samples 0,3 , and 1 , respectively. No statistically significant difference in the value of the OSI index was found among these samples. Also, no significant difference was found $(p<0.05)$ between the OSI index values of the initial samples (Figure 2a) and the samples after the frying process (Figure $2 b$ ).

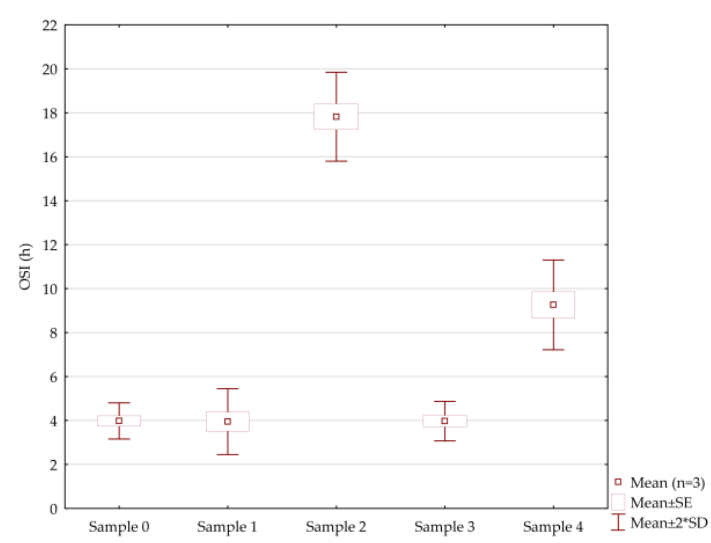

(a)

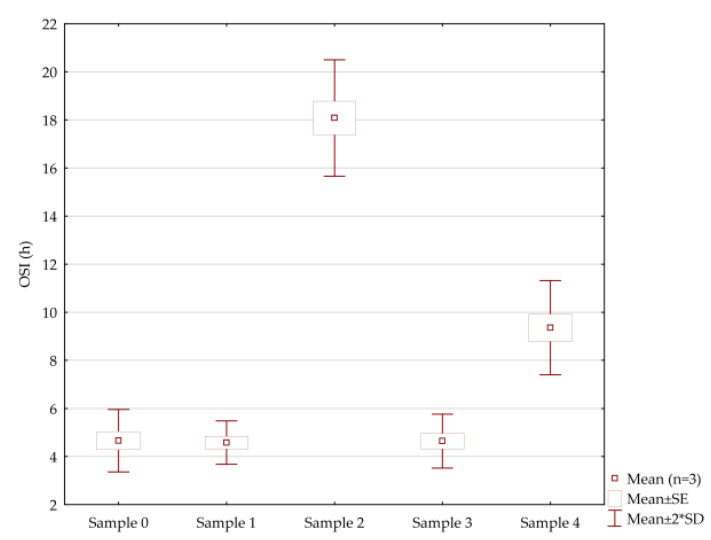

(b)

Figure 2. Box plot presented OSI index values of (a) the examined initial samples and (b) examined samples after the frying process.

The TOTOX index gives a comprehensive picture of oxidation changes as it includes both primary and secondary oxidation products. The lowest value of the TOTOX index in the initial samples was noticed in sample 4, while the highest was determined in sample 1 (Table 2). Standard sunflower oil proved to be the most oxidatively unstable after frying, so this oil is the worst choice for food frying compared with the other investigated samples. No statistically significant difference $(p<0.05)$ was found in the TOTOX index of standard sunflower oil with the addition of natural and synthetic antioxidants after frying. The addition of antioxidants to standard sunflower oil proved to be a better frying option compared to palmolein, where the TOTOX index after frying was $66.97 \pm 4.57$. However, high-oleic sunflower oil has proven to be the most oxidatively stable and the best frying oil according to the TOTOX index. The smallest increase in the TOTOX index compared to the initial sample of 4.01 times was recorded in sample 1, while the largest increase, as much as 6.65 times, was determined in sample 4. Kondratowicz-Pietruszka et al. [16] examined the values of the TOTOX index of rapeseed and coconut oil and after $300 \mathrm{~min}$ of frying obtained increases of 23.45 and 40.00 times in relation to the initial values.

Table 2. TOTOX values obtained before and after frying of examined samples. Values are means \pm standard deviation $(n=3)$; Different lower-case letters in the same column indicate significantly different values between samples while different upper-case letters in the same row indicate significantly different TOTOX values before and after frying $(p<0.05)$.

\begin{tabular}{ccc}
\hline & Before Frying & After Frying \\
\hline Sample 0 & $12.41 \pm 0.87 \mathrm{aA}$ & $80.80 \pm 3.24 \mathrm{~dB}$ \\
Sample 1 & $12.70 \pm 0.92^{\mathrm{aA}}$ & $50.92 \pm 6.12 \mathrm{aB}$ \\
Sample 2 & $7.60 \pm 0.84^{\mathrm{cA}}$ & $48.32 \pm 3.15_{\mathrm{aB}}$ \\
Sample 3 & $10.59 \pm 1.75^{\mathrm{aA}}$ & $66.97 \pm 4.57 \mathrm{cB}$ \\
Sample 4 & $4.73 \pm 0.45^{\mathrm{bA}}$ & $31.48 \pm 3.56 \mathrm{bB}$ \\
\hline
\end{tabular}


The correlation between parameters was also examined and a significant negative correlation $(p<0.05)$ of iodine value and OSI index $(-0.97)$ was obtained, while the obtained correlation of the TOTOX index with the iodine value $(0.20)$ and OSI index $(-0.14)$ was insignificant. A significant negative correlation found between the iodine value and the OSI index is also noticeable in Figures 1 and 2 and was confirmed in previous studies [17].

\section{Conclusions}

Based on the obtained results, it was concluded that the frying process does not significantly affect the iodine value of the tested samples. Chebet et al. [13] also concluded that the frying process does not significantly affect the iodine value of palm oil, while in soybean oil, frying leads to a significant reduction in iodine value. Of the tested samples, the worst choice for frying turned out to be standard sunflower oil, the most often used in the household for these purposes due to its affordability. Modification of this oil by the addition of antioxidants (natural or synthetic) showed an improvement in the oxidative properties of these oils, the TOTOX index after the frying process decreased by 32.48 and 13.83 by the addition of synthetic and natural antioxidants. A slightly better frying oil is palmolein with a TOTOX index 29.88 lower than refined sunflower oil, while the best frying oil was high-oil sunflower oil with a TOTOX index 49.32 lower compared to the control sample.

Author Contributions: Conceptualization, R.R.; methodology, R.R.; software, T.L.; formal analysis, T.L. and K.G.; investigation, T.L. and K.G.; writing-original draft preparation, T.L. and K.G.; writing - review and editing, R.R.; supervision, R.R. All authors have read and agreed to the published version of the manuscript.

Acknowledgments: This research is financed by the Ministry of Education, Science and Technology Development of the Republic of Serbia, Project Number 451-03-68/2020-14/200134.

Conflicts of Interest: The authors declare no conflict of interest.

\section{References}

1. Gupta, M.K. Frying Oils. In Bailey's Industrial Oil and Fat Products; Wiley: Hoboken, NJ, USA, 2020; pp. 1-30.

2. Ali, R.F. Improvement the stability of fried sunflower oil by using different levels of pomposia (Syzyygium cumini). Electron. J. Environ. Agric. Food Chem. 2010, 9. Available online: https://www.researchgate.net/publication/228704549_Improvement_the_stability_of_fried_sunflower_oil_by_using_different_levels_of_pomposia_Syzyygium_Cumini (accessed on 3 November 2020).

3. Raza, S.A.; Rashid, A.; William, J.; Razzaq, A. Evaluation of oxidative stability of sunflower oil at frying temperature in presence of butylated hydroxytoluene and methanolic extracts of medicinally important plants of Pakistan. Int. Food Res. J. 2014, 21, 331334.

4. Wang, D.; Chen, X.; Wang, Q.; Meng, Y.; Wang, D.; Wang, X. Influence of the essential oil of Mentha spicata cv. Henanshixiang on sunflower oil during the deep-frying of Chinese Maye. LWT 2020, 122, 109020.

5. Iqbal, S.; Haleem, S.; Akhtar, M.; Zia-ul-Haq, M.; Akbar, J. Efficiency of pomegranate peel extracts in stabilization of sunflower oil under accelerated conditions. Food Res. Int. 2008, 41, 194-200.

6. Mishra, S.K.; Belur, P.D.; Iyyaswami, R. Use of antioxidants for enhancing oxidative stability of bulk edible oils: A review. Int. J. Food Sci. Technol. 2020, doi:10.1111/ijfs.14716.

7. ISO 3961:2018. Animal and Vegetable Fats and Oils - Determination of Iodine Value; International Organization for Standardization: Geneva, Switzerland, 2016.

8. Metrohm Application Bulletin 204/2e. Oxidation Stability of Oils and Fats-Rancimat Method. pp. 1-4 Available online: https://www.metrohm.com/en/applications/AB-204, 10/23/2020 (accessed on 3 November 2020).

9. ISO 6886:2016. Animal and Vegetable Fats and Oils-Determination of Oxidative Stability (Accelerated Oxidation Test); International Organization for Standardization: Geneva, Switzerland, 2016.

10. Shahidi, F.; Zhong, H.Y.J. Methods for Measuring Lipid Oxidation. In Bailey's Industrial Oil and Fat Products, 7th ed.; Shahidi, F., Ed.; John Wiley \& Sons, Ltd.: Hoboken, NJ, USA, 2020; pp. 1-27.

11. ISO 3960: 2017. Animal and Vegetable Fats and Oils-Determination of Peroxide Value-Iodometric (visual) Endpoint Determination; International Organization for Standardization: Geneva, Switzerland, 2017.

12. ISO 6885:2016. Animal and Vegetable Fats and Oils-Determination of Anisidine Value; International Organization for Standardization: Geneva, Switzerland, 2016. 
13. Chebet, J.; Kinyanjui, T.; Cheplogoi, P. Impact of frying on iodine value of vegetable oils before and after deep frying in different types of food in Kenya. J. Sci. Innov. Res. 2016, 5, 193-196.

14. Akoh, C.C. Oxidative stability of fat substitutes and vegetable oils by the oxidative stability index method. J. Am. Oil. Chem. Soc. 1994, 71, 211-216.

15. Isbell, T.A.; Abbott, T.P.; Carlson, K.D. Oxidative stability index of vegetable oils in binary mixtures with meadowfoam oil. Ind. Crops Prod 1999, 9, 115-123.

16. Kondratowicz-Pietruszka, E.; Ostasz, L.; Tataruch, K. The dynamics of oxidative changes in selected fats during the frying of French fries. Acta Sci. Pol. Technol. Aliment. 2019, 18, 293-303.

17. Górnaś, P.; Rudzińska, M.; Soliven, A. Industrial by-products of plum Prunus domestica L. and Prunus cerasifera Ehrh. as potential biodiesel feedstock: Impact of variety. Ind. Crops Prod. 2017, 100, 77-84. 